\title{
UNIQUE FIXED POINT THEOREM FOR WEAKLY C-CONTRACTIVE MAPPINGS
}

\author{
Binayak S. Choudhury \\ Department of Mathematics \\ Bengal Engineering and Science University, Shibpur \\ P.O. - B. Garden, Shibpur \\ Howrah - 711103, West Bengal, INDIA \\ Corresponding Author: bsc@math.becs.ac.in \\ Received 11 August, 2008; Revised 30 September, 2008
}

\begin{abstract}
In this work we introduce the class of weakly c-contractive mappings. We establish that these mappings necessarily have unique fixed points in complete metric spaces. We support our result by an example. Our result also generalises an existing result in metric spaces.
\end{abstract}

Key words : Metric space, Fixed point, Weak $C$-contraction.

M S C (2000) : 54H25

\section{INTRODUCTION}

It is well known that Banach's contraction mapping theorem is one of the pivotal results of functional analysis. A mapping $T: X \rightarrow X$ where $(X, d)$ is a metric space, is said to be a contraction if there exists $0<k<1$ such that for all $x, y \in X$,

$$
d(T x, T y) \leq k d(x, y)
$$

If the metric space $(X, d)$ is complete then the mapping satisfying (1.1) has a unique fixed point. Inequality (1.1) implies continuity of $T$. A natural question is that whether we can find contractive conditions which will imply existence of fixed point in a complete metric space but will not imply continuity.

Kannan $[10,11]$ established the following result in which the above question has been answered in the affirmative.

If $T: X \rightarrow X$ where $(X, d)$ is a complete metric space, satisfies the inequality

$$
d(T x, T y) \leq k[d(x, T x)+d(y, T y)]
$$


where $0<k<\frac{1}{2}$ and $x, y \in X$, then $T$ has a unique fixed point.

The mapping $T$ need not be continuous as has been established through examples[11]. The mappings satisfying (1.2) are called Kannan type mappings. There is a large literature dealing with Kannan type mappings and their generalization some of which are noted in [8], [17] and [19].

A similar contractive condition has been introduced by Chatterjee [6]. We call this contraction a $C$-contraction (borrowing the name from the name of its author).

Definition $1.1 C$-contraction [6].

Let $T: X \rightarrow X$ where $(X, d)$ is a metric space is called a $C$-contraction if there exists $0<k<\frac{1}{2}$ such that for all $x, y \in X$ the following inequality holds:

$$
d(T x, T y) \leq k[d(x, T y)+d(y, T x)]
$$

Theorem 1.1 [6] A $C$-contraction defined on a complete metric space has a unique fixed point.

In establishing theorem 1.1 there is no requirement of continuity of the $C$-contraction.

It has been established in [15] that inequalities (1.1), (1.2) and (1.3) are independent of one another. $C$-contraction and its generalizations have been discussed in a number of works some of which are noted in [4], [8], [9] and [19].

Banach's contraction mapping theorem has been generalized in a number of recent papers. As for example, asymptotic contraction has been introduced by Kirk [12] and generalized Banach contraction conjecture has been proved in [1] and [14].

Particularly a weaker contraction has been introduced in Hilbert spaces in [2]. The following is the corresponding definition in metric space.

\section{Definition 1.2 Weakly contractive mapping}

A mapping $T: X \rightarrow X$ where $(X, d)$ is a complete metric space is said to be weakly

contractive if $\quad d(T x, T y) \leq d(x, y)-\psi(d(x, y))$,

where $x, y \in X, \psi:[0, \infty) \rightarrow[0, \infty)$ is continuous and nondecreasing,

$\psi(x)=0$ if and only if $x=0$ and $\lim _{x \rightarrow \infty} \psi(x)=\infty$.

If we take $\psi(x)=k x$ where $0<k<1$ then (1.4) reduces to (1.1). 
There are a number of works in which weakly contractive mappings have been considered. Some of these works are noted in [3], [7], [13], and [16].

In the present work in the same spirit we introduce a generalization of $C$-contraction.

\section{Definition 1.3 Weak C-contraction :}

A mapping $T: X \rightarrow X$, where $(X, d)$ is a metric space is said to be weakly $C$-contractive or a weak C-contraction if for all $x, y \in X$,

$$
d(T x, T y) \leq \frac{1}{2}[d(x, T y)+d(y, T x)]-\psi(d(x, T y), d(y, T x))
$$

where $\psi:[0, \infty)^{2} \rightarrow[0, \infty)$ is a continuous mapping such that $\psi(x, y)=0$ if and only if $x=y=0$.

If we take $\psi(x, y)=k(x+y)$ where $0<k<\frac{1}{2}$ then (1.5) reduces to (1.4), that is weak $\mathrm{C}$-contractions are generalisations of $\mathrm{C}$-contractions. At the end of the next section we discuss an example which shows that weak C-contractions constitute a strictly larger class of mappings than $\mathrm{C}$-contractions.

In the next section we establish that in a complete metric space a weak C-contraction has a unique fixed point.

\section{MAIN RESULTS}

Theorem 2.1 Let $T: X \rightarrow X$, where $(X, d)$ is a complete metric space be a weak Ccontraction. Then $T$ has a unique fixed point.

\section{Proof}

Let $x_{0} \in X$ and for all $n \geqq 1, \quad x_{n+1}=T x_{n}$.

If $x_{n}=x_{n+1}=T x_{n}$ then $x_{n}$ is a fixed point of $T$.

So we assume $x_{n} \neq x_{n+1}$.

Putting $x=x_{n-1}$ and $y=x_{n}$ in $(1.5)$ we have for all $\mathrm{n}=0,1,2, \ldots \ldots$

$$
\begin{aligned}
& d\left(x_{n}, x_{n+1}\right)=d\left(T x_{n-1}, T x_{n}\right) \\
& \leq \frac{1}{2}\left(d\left(x_{n-1}, T x_{n}\right)+d\left(x_{n}, T x_{n-1}\right)\right)-\psi\left(d\left(x_{n-1}, T x_{n}\right), d\left(x_{n}, T x_{n-1}\right)\right) \\
& =\frac{1}{2} d\left(x_{n-1}, x_{n+1}\right)-\psi\left(d\left(x_{n-1}, x_{n+1}\right), 0\right) \\
& \leq \frac{1}{2}\left(d\left(x_{n-1}, x_{n}\right)+d\left(x_{n}, x_{n+1}\right)\right)-\psi\left(d\left(x_{n-1}, x_{n+1}\right), 0\right)
\end{aligned}
$$


From (2.2), for all $n=1,2, \ldots \ldots$

$$
d\left(x_{n}, x_{n+1}\right) \leq d\left(x_{n-1}, x_{n}\right)
$$

Thus $\left\{d\left(x_{n}, x_{n+1}\right)\right\}$ is a monotone decreasing sequence of non-negative real numbers and hence is convergent.

Let $d\left(x_{n}, x_{n+1}\right) \rightarrow r$ as $n \rightarrow \infty$.

We next prove that $r=0$.

$$
\begin{aligned}
& d\left(x_{n}, x_{n+1}\right)=d\left(T x_{n-1}, T x_{n}\right) \\
& \quad \leq \frac{1}{2}\left(d\left(x_{n-1}, T x_{n}\right)+d\left(x_{n}, T x_{n-1}\right)\right)-\psi\left(d\left(x_{n-1}, x_{n+1}\right), d\left(x_{n}, x_{n}\right)\right) \quad(\text { by }(1.5)) \\
& \quad \leq \frac{1}{2} d\left(x_{n-1}, x_{n+1}\right) \\
& \quad \leq \frac{1}{2}\left(d\left(x_{n-1}, x_{n}\right)+d\left(x_{n}, x_{n+1}\right)\right) .
\end{aligned}
$$

Making $n \rightarrow \infty$ we have by (2.4),

$$
\begin{aligned}
& r \leq \lim _{n \rightarrow \infty} \frac{1}{2} d\left(x_{n-1}, x_{n+1}\right) \leq \frac{1}{2} r+\frac{1}{2} r, \\
& \text { or, } \lim _{n \rightarrow \infty} d\left(x_{n-1}, x_{n+1}\right)=2 r .
\end{aligned}
$$

Making $n \rightarrow \infty$ in (2.2) and using (2.4), (2.5) and the continuity of $\psi$ we have $r \leq r-\psi(2 r, 0)$.

or, $\psi(2 r, 0) \leq 0$, which is a contradiction unless $r=0$.

Thus we have established that $d\left(x_{n}, x_{n+1}\right) \rightarrow 0$ as $n \rightarrow \infty$.

Next we show that $\left\{x_{n}\right\}$ is a cauchy sequence. If otherwise, then there exist $\epsilon>0$ and increasing sequences of integers $\{m(k)\}$ and $\{n(k)\}$ such that for all integers $k$,

$$
\begin{aligned}
& n(k)>m(k)>k, \\
& d\left(x_{m(k)}, x_{n(k)}\right) \geq \epsilon, \text { and }
\end{aligned}
$$




$$
d\left(x_{m(k)}, x_{n(k)-1}\right)<\epsilon
$$

Then,

$$
\begin{aligned}
& \epsilon \leq d\left(x_{m(k)}, x_{n(k)}\right) \\
& =d\left(T x_{m(k)-1}, T x_{n(k)-1}\right) \\
& \leq \frac{1}{2}\left(d\left(x_{m(k)-1}, T x_{n(k)-1}\right)+d\left(x_{n(k)-1}, T x_{m(k)-1}\right)\right) \\
& -\psi\left(d\left(x_{m(k)-1}, T x_{n(k)-1}\right), d\left(x_{n(k)-1}, T x_{m(k)-1}\right)\right) \quad(b y(1.5)) \\
& =\frac{1}{2}\left(d\left(x_{m(k)-1}, x_{n(k)}\right)+d\left(x_{n(k)-1}, x_{m(k)}\right)\right) \\
& -\psi\left(d\left(x_{m(k)-1}, x_{n(k)}\right), d\left(x_{n(k)-1}, x_{m(k)}\right)\right) .
\end{aligned}
$$

Again,

$$
\begin{aligned}
& \epsilon \leq d\left(x_{m(k)}, x_{n(k)}\right) \\
& \left.\leq d\left(x_{m(k)}, x_{n(k)-1}\right)+d\left(x_{n(k)-1}, x_{n(k)}\right)\right) \\
& \leq \epsilon+d\left(x_{n(k)-1}, x_{n(k)}\right) . \quad(\text { by }(2.9))
\end{aligned}
$$

Making $k \rightarrow \infty$ in the above inequality and using (2.6)we obtain

$$
\lim _{k \rightarrow \infty} d\left(x_{m(k)}, x_{n(k)}\right)=\epsilon
$$

and $\quad \lim _{k \rightarrow \infty} d\left(x_{m(k)}, x_{n(k)-1}\right)=\epsilon$.

Again,

$$
\begin{aligned}
d\left(x_{m(k)}, x_{n(k)-1}\right) & \leq d\left(x_{m(k)}, x_{m(k)-1}\right) \\
& +d\left(x_{m(k)-1}, x_{n(k)}\right) \\
& +d\left(x_{n(k)}, x_{n(k)-1}\right)
\end{aligned}
$$

Also, $\quad d\left(x_{m(k)-1}, x_{n(k)}\right) \leq d\left(x_{m(k)-1}, x_{m(k)}\right)+d\left(x_{m(k)}, x_{n(k)}\right)$.

Making $k \rightarrow \infty$ in the above two inequalities and using (2.6), (2.11) and (2.12) we get,

$$
\lim _{k \rightarrow \infty} d\left(x_{m(k)-1}, x_{n(k)}\right)=\epsilon
$$


Next making $k \rightarrow \infty$ in (2.10) and using (2.6), (2.12) and (2.13) we obtain

$$
\epsilon \leq \frac{1}{2}(\epsilon+\epsilon)-\psi(\epsilon, \epsilon)
$$

or, $\psi(\epsilon, \epsilon) \leq 0$, which is a contradiction since $\epsilon>0$. Hence $\left\{x_{n}\right\}$ is a cauchy sequence and therefore is convergent in the complete metric space $(X, d)$.

Let $x_{n} \rightarrow z$ as $n \rightarrow \infty$.

Then, $d(z, T z) \leq d\left(z, x_{n+1}\right)+d\left(x_{n+1}, T z\right)$

$$
\begin{aligned}
& \leq d\left(z, x_{n+1}\right)+d\left(T x_{n}, T z\right) \\
& \leq d\left(z, x_{n+1}\right)+\frac{1}{2}\left(d\left(z, T x_{n}\right)+d\left(x_{n}, T z\right)\right)-\psi\left(d\left(z, T x_{n}\right), d\left(x_{n}, T z\right)\right) \\
& =d\left(z, x_{n+1}\right)+\frac{1}{2}\left(d\left(z, x_{n+1}\right)+d\left(x_{n}, T z\right)\right)-\psi\left(d\left(z, x_{n+1}\right), d\left(x_{n}, T z\right)\right)
\end{aligned}
$$

Making $n \rightarrow \infty$, using (2.14) and continuity of $\psi$ we obtain

$$
\left.d(z, T z) \leq \frac{1}{2} d(z, T z)-\psi(0, d(z, T z)) \leq \frac{1}{2} d(z, T z)\right)
$$

which is a contradiction unless $d(z, T z)=0$.

Hence $z=T z$.

Next we establish that the fixed point $z$ is unique.

If $z_{1}$ and $z_{2}$ are two fixed points of $T$, then

$$
d\left(z_{1}, z_{2}\right)=d\left(T z_{1}, T z_{2}\right) \leq \frac{1}{2}\left(d\left(z_{1}, T z_{2}\right)+d\left(z_{2}, T z_{1}\right)\right)-\psi\left(d\left(z_{1}, T z_{2}\right), d\left(z_{2}, T z_{1}\right)\right) .
$$

that is, $d\left(z_{1}, z_{2}\right) \leq d\left(z_{1}, z_{2}\right)-\psi\left(d\left(z_{1}, z_{2}\right), d\left(z_{1}, z_{2}\right)\right)$,

which by property of $\psi$ is a contradiction unless $d\left(z_{1}, z_{2}\right)=0$, that is $z_{1}=z_{2}$. This completes the proof of the uniqueness of the fixed point.

We next consider the following example.

Example 2.1: Let $X=\{\alpha, \beta, \gamma\}$ and $d$ is a metric defined on $X$ as follows. $d(\alpha, \beta)=1, \quad d(\beta, \gamma)=2, \quad d(\gamma, \alpha)=1.5$

Then $(X, d)$ is a complete metric space. Let $\psi(a, b)=\frac{1}{2} \min \{a, b\}$. 
Let $T: X \rightarrow X$ be a mapping defined as follows.

$T \alpha=\beta, T \beta=\beta, T \gamma=\alpha$.

Then $T$ is a weak C-contraction and conditions of theorem 2.1 are satisfied. Hence $T$ must have a unique fixed point. Here $\beta$ is the unique fixed point of $T$.

The mapping $T$ in the above example is not a C-contraction. This is seen by noting that when $x=\alpha$ and $y=\gamma$ the inequality (1.3)will not be satisfied by $T$. Again as has been noted in the discussion following definition 1.3, every C-contraction is a weak $\mathrm{C}$-contraction. Thus the class of weakly $\mathrm{C}$-contractive mappings is actually a strictly larger class of mappings than the class of C-contractions.

\section{REFERENCES}

1. Arvanitakis.A.D, A proof of the generalized Banach contraction conjecture, Proc. Amer. Math. Soc., 131(12), 2003, 3647 - 3656.

2. Alber.Ya.I and Guerre-Delabrieer.S, Principles of weakly contractive maps in Hilbert spaces, new results in operator theory,Advances and Appl.

(ed.by I. Gohberg and Yu Lyubich), Birkhauser Verlag, Basel 98 1997, 7 - 22.

3. Beg.I and Abbas.M, Coincidence point and Invariant Approximation for mappings satisfying generalized weak contractive condition, Fixed Point Th. and Appl., 2006, Article ID 74503, 1-7.

4. Berinde.V, Error estimates for approximating fixed points of quasi contractions, General Mathematics, 13, 2005, 23-34.

5. Berinde.V, Picard Iteration converges faster than Mann Iteration for a class of quasi contractive operators, Fixed Point Theory and Applications 2004, 97-105.

6. Chatterjea.S.K, Fixed point theorems, C. R. Acad. Bulgare Sci. , 25 , 1972 , 727 730 .

7. Chidume.C.E, H.Zegeye and S.J.Aneke, Approximation of fixed points of weakly contractive nonself maps in Banach spaces, J.Math.Anal.Appl. 270, 2002, 189-199.

8. Ghosh.K.M, A generalization of contraction principle, Internat. J.Math. and Math. Sci., Vol.4, No.1 1981, 201-207.

9. Gu.F, Strong convergence of an explicit iterative process with mean errors for a finite family of Ciric quasi contractive operators in normed spaces, Mathematical Communications, 12,2007, 75-82.

10. Kannan.R, Some results on fixed points, Bull.Calcutta Math.Soc., 60, 1968, 71-76. 
11. Kannan.R, Some results on fixed points -II, Amer.Math.Monthly, 76, 1969,405-408.

12. Kirk.W.A, Fixed points of asymptotic contraction, J.Math. Anal. Appl., 277, 2003, 645-650.

13. Mai.J.H and Liu.X.H, Fixed points of Weakly contractive Maps and Boundedness of orbits, Fixed Point Theory and Applications, 2007, Article ID 20962.

14. Merryfield.J, Rothschild.B and Stein Jr. J. D, An application of Ramsey's theorem to the Banach Contraction Principle, Proc. Amer. Math. Soc., 130 , 2002,927-933.

15. Rhoades.B.E, A comparison of various definitions of contractive mappings, Trans.Amer.Math.Soc. 226, 1977, 257-290.

16. Rhoades.B.E, Some theorems on weakly contractive maps, Nonlinear Analysis, 47 , 2001, 2683-2693.

17. Shioji.N, Suzuki.T and Takahashi.W, Contractive mappings, Kannan Mappings and Metric Completeness, Proc. Amer .Math .Soc. , 126, 1998, 3117-3124.

18. Suzuki.T, Several fixed point theorems concerning $\tau$ - distance, Fixed Point Theory and Applications , 2004(2004), 195-209.

19. Zamfirescu.T, Fix point theorems in Metric spaces , Arch. Math. , 23, 1972, 292-298. 